\title{
Optical Sensitivity of TL Glow Peaks Separated Using Computerized Glow Curve Deconvolution for RTL
} Quartz

\author{
Myung-Jin Kim', Ki-Bum Kim², Duk-Geun Hong ${ }^{3, *}$ \\ ${ }^{1}$ Archaeological Science Institute, RADPION Inc., Daejeon, Korea; ${ }^{2}$ Institute of Liberal Education, Kangwon National University, Chuncheon, Korea; \\ ${ }^{3}$ Department of Physics, Kangwon National University, Chuncheon, Korea
}

\section{Original Research}

Received July 25, 2018

Revision September 5, 2018

Accepted September 27, 2018

Corresponding author: Duk-Geun Hong

Department of Physics, Kangwon National University, 1 Gangwondaehakgil, Chuncheon 24341, Korea

Tel: $+82-33-250-8473$

Fax: +82-33-250-5666

E-mail:dghong@kangwon.ac.kr

This is an Open-Access article distributed under the terms of the Creative Commons Attribution NonCommercial License (http://creativecommons.org/ licenses/by-nc/4.0) which permits unrestricted noncommercial use, distribution, and reproduction in any medium, provided the original work is properly cited.

Copyright $\odot$ 2018 The Korean Association for Radiation Protection

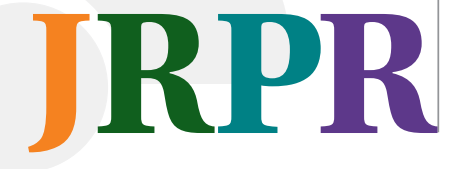

Background: The retrospective dosimetry using RTL quartz can be improved by information for an optical sensitivity of sample connected with the equivalent dose determination.

Materials and Methods: The quartz sample from a volcanic rock of Japan was used. After correcting the thermal quenching effect, RTL peaks of quartz were separated by the CGCD method cooperated with the general order kinetics. The number of overlapped glow peaks were ascertained by the $T_{m}-T_{\text {stop }}$ method. The optical sensitivity was examined by comparing the change of intensity on RTL glow peaks measured after exposure to light from a solar simulator with various illumination times.

Results and Discussion: Seven glow peaks appeared to be overlapped on the RTL glow curve. The general order kinetics model was appropriate to separate glow peaks. After exposure to light from a solar simulator from a few minutes to $416 \mathrm{hr}$, the signals for peaks P4 and P5 decayed following the form of $f(t)=a_{1} e^{-\lambda 1 t}$, while the signals for peaks P6 and P7 decayed by the form of $f(t)=a_{1} e^{-\lambda_{1} t}+a_{2} e^{-\lambda_{2} t}+a_{3} e^{-\lambda_{3} t}$.

Conclusion: For dosimetric peaks, the times taken to reduce the RTL signal to half of its initial value were $70 \mathrm{sec}$ for the peak P4, $54 \mathrm{~s}$ for the peak P5, 9,840 sec for the peak P6 and 26,580 sec for the peak P7, respectively. We conclude that the optical sensitivity of peaks P4, and P5 gives much higher than that of peaks P6 and P7.

Keywords: Optical sensitivity, RTL quartz, Thermal quenching, General order kinetics, Computerized glow curve deconvolution

\section{Introduction}

It is well known that the colours of radiation induced thermoluminescence (TL) of natural quartz typically indicate blue with an emission wavelength of around $470 \mathrm{~nm}$ and red with an emission wavelength of around $630 \mathrm{~nm}$. The quartz annealed at high temperature, such as volcanically originated tephra layers or archaeological heated materials, shows a red TL (RTL), whereas the quartz from sediment emits a blue TL (BTL). Of two types of quartz, the RTL quartz gives some advantages in the field of retrospective dosimetry, due to much higher saturation response to radiation dose than BTL quartz and stability of the TL signal during the long timescale. Extensive research has been performed to explore the RTL characteristics [1,2]. The methodological tech- 
nique for the retrospective dosimetry can be improved by information for an optical sensitivity of sample, so called bleaching, relevant to the determination of the equivalent dose. Concerning the optical sensitivity, Scholefield and Prescott et al. [3] reported that by optical stimulation, the peak at $350^{\circ} \mathrm{C}$ of the natural RTL quartz reduced slowly while a rapid decrease was found in the peaks at $270^{\circ} \mathrm{C}$ and $305^{\circ} \mathrm{C}$ of some RTL quartz. Fattahi and Stokes [2] also demonstrated that natural RTL quartz was bleachable by sunlight. Later, Lai and Murray [4] found that after exposure to sunlight, the TL intensity of RTL quartz from Chinese loess was reduced but up to approximately $40 \%$ of the natural signal did not show any change. Such exploitation by the similar approach has been extensively investigated on many kinds of quartz. However, most work was carried out with the TL signal integrated for a specific temperature limits of interest in the glow curve. However, it has been suggested that the glow curve of the quartz sample is overlapped with several peaks $[5,6]$. Although various techniques have been introduced to separate TL glow curve, the peak separation using the computerized glow curve deconvolution (CGCD) method has some advantages in the instance of the glow curve overlapped with several glow peaks [7].

In this work, we investigated on the optical sensitivity using a solar simulator of TL peaks separated by the CGCD method for RTL quartz of volcanic origin from Japan.

\section{Materials and Methods}

The quartz sample collected from a volcanic rock in Iwate Prefecture (Japan) was employed for this study. Isolation of the quartz was made by density between 2.60 and $2.70 \mathrm{~g} \cdot \mathrm{cm}^{-3}$. The quartz was treated with $40 \%$ hydrofluoric acid for 1 hour to eliminate the feldspar and then rinsed with $10 \% \mathrm{H}_{2} \mathrm{O}_{2}$ and $10 \% \mathrm{HCl}$. The resulting quartz was sieved into grain size of $\sim 125 \mathrm{~mm}$ and the purity of quartz sample was checked by measuring the luminescence by infrared stimulation.

An automated Riso TL/OSL system (Model TL/OSLDA-15) in the Central Laboratory of Kangwon National University was employed for all RTL measurements. Irradiation to samples was made with a ${ }^{90} \mathrm{Sr} /{ }^{90} \mathrm{Y}$ beta source delivering approximately $0.13 \mathrm{~Gy} \cdot \mathrm{s}^{-1}$, which was calibrated using a standard quartz, housed in the system. The detection of RTL signal was performed by a PMT (R943-01 Hamamatsu Photonics Corp., Shizuoka, Japan) attached with a Cormar 650IU band-pass filter with a transmission range of 630-670 nm.
The time independent signal was reduced using a thermoelectric refrigeration chamber (PC182CE PHOTOCOOL) maintained at $20^{\circ} \mathrm{C}$.

\section{Results and Discussion}

\section{Emission property of RTL quartz sample}

The emission wavelength of RTL quartz sample used in this study was tested by the spectral analysis using an on-line three-dimensional TL spectrometer [8]. The RTL quartz sample preheated for $60 \mathrm{~s}$ at $220^{\circ} \mathrm{C}$ after an X-ray exposure of about $2 \mathrm{kGy}$ was prepared for this test. As shown in Figure 1, the spectrum clearly shows a strong emission peak in a wavelength of around $660 \mathrm{~nm}$, peaking at $\sim 350^{\circ} \mathrm{C}$.

\section{Thermal quenching}

Prior to the peak separation, the checking of thermal quenching is an important process because the luminescence efficacy to radiation may change with temperature. For checking of the thermal quenching, RTL measurements were made for heating rates changing from $0.5^{\circ} \mathrm{C} \cdot \mathrm{s}^{-1}$ to $10^{\circ} \mathrm{C} \cdot \mathrm{s}^{-1}$ with the samples exposed with a beta dose of $300 \mathrm{~Gy}$ as shown in Figure 2. If the thermal quenching does not occur, the TL glow peak area is anticipated to be constant irrespective of the variation of heating rate. However, we observed that the area integrated at the higher temperature peaks on the glow curve clearly reduced according to the increment of heating rate, as shown in Figure 2. This suggests

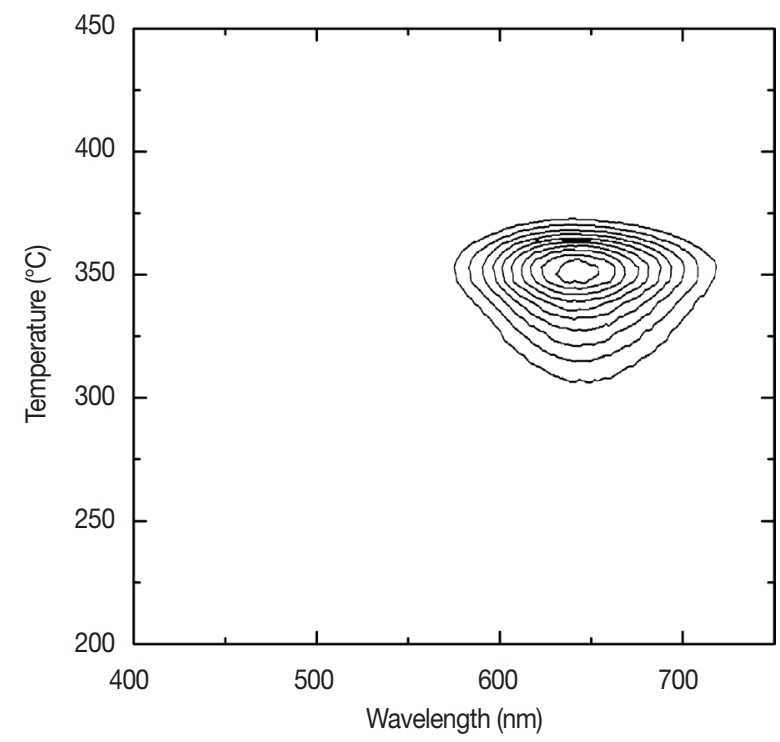

Fig. 1. Three dimensional emission spectrum for the RTL quartz sample used in this study. 


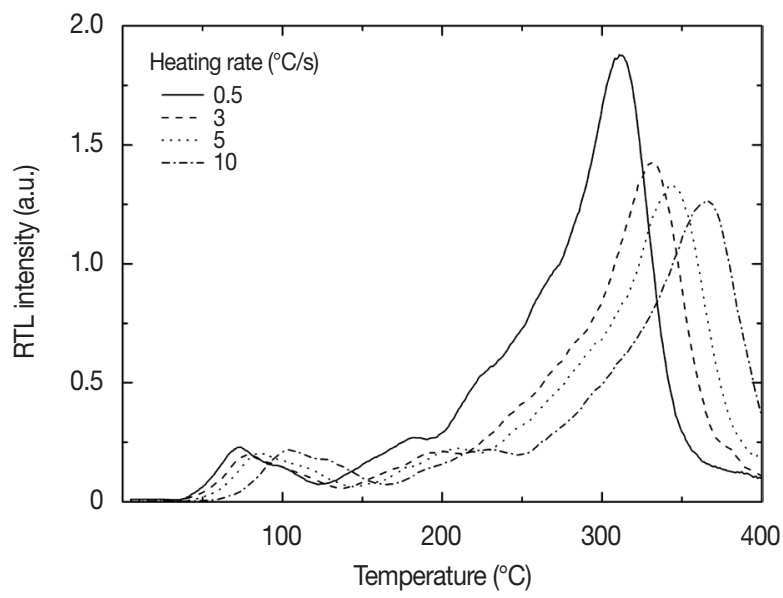

Fig. 2. RTL glow curves measured for different heating rates.

that the thermal quenching took place [9]. In this study we corrected the thermal quenching by means of the normalisation of RTL intensity measured for various heating rates [9]. The effect of thermal quenching is represented by the thermal quenching efficiency given by

$$
\eta(T)=\frac{1}{1+C \exp \left(-\frac{W}{k_{b} T}\right)}
$$

where the values of $W, C, k_{b}$ and $T$ mean the thermal activation energy for thermal quenching, a constant, the Boltzmann constant and the temperature, respectively. The values of $W$ and $C$ are produced from the luminescence efficiency of the RTL peaks for various heating rates (Figure 3). From this procedure, the resultant values of $C$ and $W$ were $0.65 \times 10^{6}$ and $0.57 \mathrm{eV}$. These values are of similar to those of other RTL quartz reported in previous [10]. The thermal quenching was then corrected by

$$
I_{Q}(T)=\eta(T) I_{U}(T)
$$

where $I_{Q}(T)$ is the RTL intensity after correcting the thermal quenching and $I_{U}(T)$ is the RTL intensity before correcting the thermal quenching.

\section{Peak separation}

In general, the glow curves of RTL quartz are overlapped with several peaks and can be separated by a CGCD method which is a very useful technique to obtain the information of trap in many instances. However, before the process of separation of glow curves using the CGCD method, it is essential to know the number of glow peaks overlapped on the glow

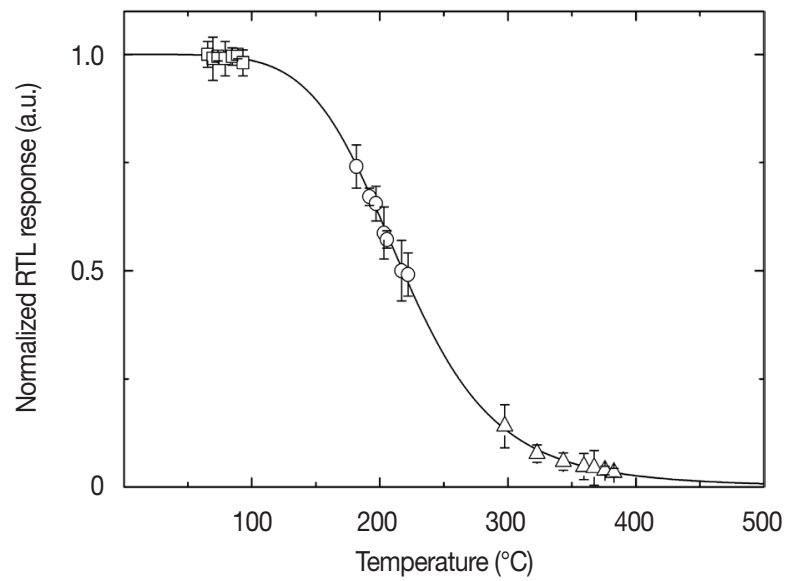

Fig. 3. Luminescence efficiency according to temperature.

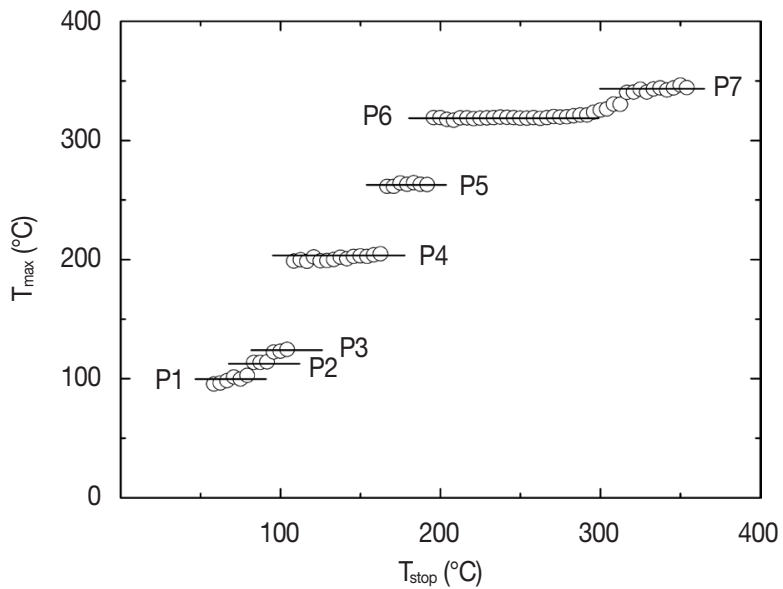

Fig. 4. First maximum temperatures $\left(T_{m}\right)$ of remaining $T L$ glow peaks versus each initial heating temperature $\left(T_{\text {stop }}\right)$.

curve. In this investigation, the number of glow peaks overlapped on the TL glow curve was identified using $T_{m}-T_{\text {stop }}$ method [11]. The procedure adopted in this study is as follows. With the quartz samples exposed with a beta dose of $300 \mathrm{~Gy}$, RTL measurement involving a partial heating was made to a temperature named as $\mathrm{T}_{\text {stop }}$ and then the samples were cooled to room temperature. This sequence is continued several times to all of the remaining glow curve at various temperatures ( $\mathrm{T}_{\text {stop }}$ ) up to $450^{\circ} \mathrm{C}$ with a $5^{\circ} \mathrm{C}$ interval. Figure 4 shows the first maximum temperatures $\left(\mathrm{T}_{\mathrm{m}}\right)$ of remaining RTL glow curves for each initial heating temperature $\left(T_{\text {stop }}\right)$. As a result, seven plateaus were found, which suggests that the RTL glow curve is overlapped with seven glow peaks.

With those information, we analysed the glow peaks to procure the trap parameters of the each peak using the CGCD method combined with the general order kinetics 


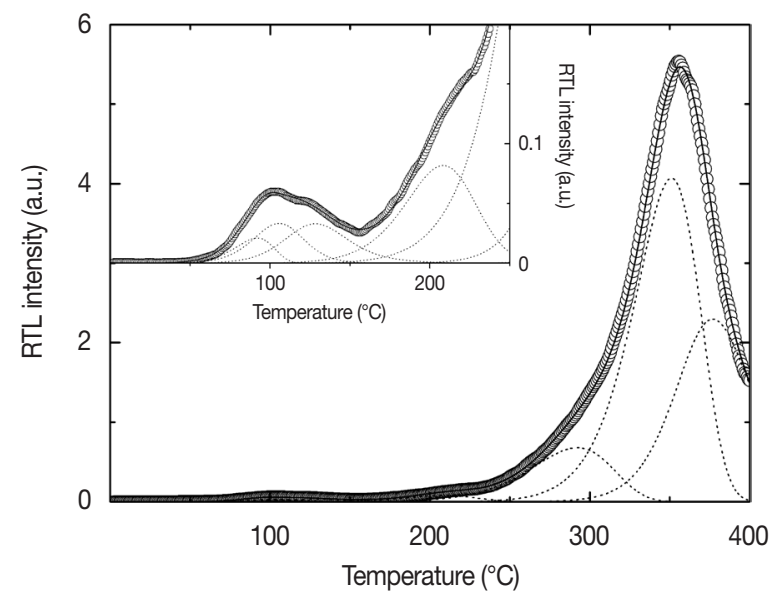

Fig. 5. RTL glow peak separated after correcting the thermal quenching.

[12]. The general order kinetics used is expressed by

$I(T)=I_{m} \frac{b}{b-1} \exp \left(\frac{E}{k_{b} T} \frac{T-T_{m}}{T_{m}}\right)\left[(b-1)(1-\Delta) \frac{T^{2}}{T_{m}^{2}} \exp \left(\frac{E}{k_{b} T} \frac{T-T_{m}}{T_{m}}\right)+Z_{m}\right]^{-\frac{b}{b-1}}$,

where $\Delta=2 k_{b} T / E, \Delta_{m}=2 k_{b} T_{m} / E, Z_{m}=1+(b-1) \Delta_{m}, I$ is the intensity of glow peak, $E$ is the activation energy, $k_{b}$ is the Boltzmann constant, $T$ is the absolute temperature and $b$ is the order of the kinetics. In this approach, the values of $E, T$, and $b$ were individually obtained by repeated fitting processes. The Mathematica software (version 9.0) was used for this analysis. Figure 5 shows the separated glow peaks after correcting the thermal quenching. The RTL glow curves were resolved into seven glow peaks centered at 92, 106, 128, 208, 292,351 , and $377^{\circ} \mathrm{C}$, listed as $\mathrm{P} 1-\mathrm{P} 7$, respectively. The activation energies and the order of kinetics determined for individual peaks are represented in Table 1 . The good-of-fit for the separated glow curves was tested by the figure of merit (FOM) [13]:

$$
\mathrm{FOM}=\frac{\sum\left|Y_{i}-f_{i}\right|}{\sum f_{i}} \times 100(\%)
$$

In this equation, $Y_{i}$ is the experimental value and $f_{i}$ is the best-fit value of the TL intensity. Balian and Eddy [13] recommend $2.5 \%$ to declare a fit as good. The FOM value obtained was $1.5 \%$, which indicates that the fit is successful.

\section{Optical sensitivity}

The optical sensitivity for the RTL quartz sample was examined by comparing the change of intensity on RTL glow peaks measured after exposure to light from a solar simulator (SOL-2: Honle, Martinsried, Germany) with various illu-
Table 1. Activation Energies (E) and Order of Kinetics (b) of the RTL Quartz Determined after Correcting the Thermal Quenching

\begin{tabular}{lccccccc}
\hline & P1 & P2 & P3 & P4 & P5 & P6 & P7 \\
\hline E(eV $)$ & 0.85 & 0.94 & 0.98 & 1.06 & 1.27 & 1.64 & 2.04 \\
$b$ & 1.04 & 1.57 & 1.97 & 1.25 & 1.00 & 1.04 & 1.99
\end{tabular}

mination times. According to the manufacturer's specification the solar simulator used in the present work provides a power density $910 \mathrm{~W} \cdot \mathrm{m}^{-2}$ at the sample position and about 6.5 times the brightness compared with sunlight. Following experiments were carried out for this investigation. After exposure with a beta dose of $300 \mathrm{~Gy}$, the RTL measurements of the quartz sample were made up to $450^{\circ} \mathrm{C}$ with the samples exposed to various times to light from a solar simulator, from a few minutes to 416 hours. With these glow curves, the thermal quenching was corrected and then peak separation using the CGCD method mentioned earlier was made. Variations of the luminescence efficacy between samples were normalized using the $120^{\circ} \mathrm{C}$ peak intensity after giving a second monitor beta dose. Three aliquots in each measurement were employed. In this work, the four peaks (P4-P7) are of interest in retrospective dosimetry because the three peaks (P1-P3) possess a short life time. As a result of the decay modes for exposure to light from a solar simulator, the signals for peaks P4 and P5 exhibited one exponential fall represented by the functional form of $f(t)=a_{1} e^{-\lambda_{1} t}$, while three different exponential decays expressed the functional form of $f(t)=a_{1} e^{-\lambda_{1} t}+a_{2} e^{-\lambda_{2} t}+a_{3} e^{-\lambda_{3} t}$ were shown for the signals for peaks $\mathrm{P} 6$ and $\mathrm{P} 7$.

The decay modes of the peaks $\mathrm{P} 4$ and P5 are shown in Figure 6A. During an exposure to light of 416 hours, a rapid initial decaying occurred, showing an exponential fall with time with a decay constant of $\lambda_{1}=5.05 \times 10^{-1} \mathrm{~min}^{-1}$ for the peak $\mathrm{P} 4$ and $7.33 \times 10^{-1} \mathrm{~min}^{-1}$ for the peak P5. Figure $6 \mathrm{~B}$ shows the decay modes of the peaks P6 and P7. There is a rapid initial decaying and the decaying keeps more slowly with two different decay shapes, showing exponential fall with time with three decay constants of $\lambda_{1}=6.96 \times 10^{-2} \mathrm{~min}^{-1}, \lambda_{2}=1.29 \times 10^{-3}$ $\min ^{-1}$ and $\lambda_{3}=6.85 \times 10^{-5} \mathrm{~min}^{-1}$ for the peak $\mathrm{P} 6$, and $\lambda_{1}=1.30 \times$ $10^{-2} \mathrm{~min}^{-1}, \lambda_{2}=1.23 \times 10^{-3} \mathrm{~min}^{-1}$ and $\lambda_{3}=7.74 \times 10^{-5} \mathrm{~min}^{-1}$ for the peak P7. The decay constants are calculated using leastsquares fits on the plot of linear-log scale and are displayed in Table 2. The times taken to reduce the RTL signal to half of its initial value were 70 seconds for the peak $\mathrm{P} 4,54$ seconds for the peak P5, 9,840 seconds for the peak P6 and 26,580 seconds for the peak P7, respectively. Scholefield and Prescott 

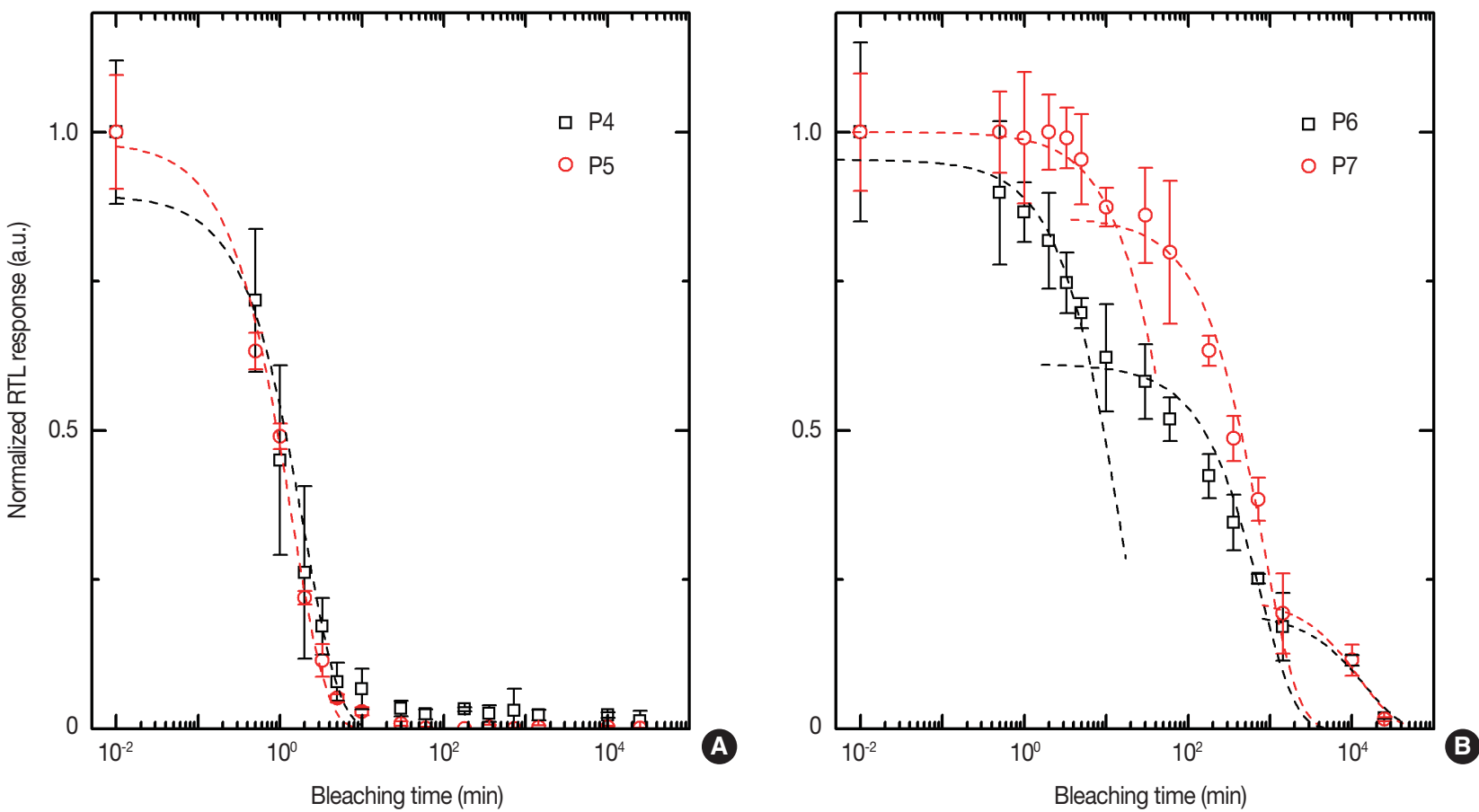

Fig. 6. Normalized RTL responses according to various exposure times to light from a solar simulator of peaks (A) P4 and P5, (B) P6 and P7.

Table 2. Decay Constants Determined for Each Glow Peak

\begin{tabular}{lccc}
\hline Peak & $\lambda_{1}$ & $\lambda_{2}$ & $\lambda_{3}$ \\
\hline P4 & $5.05 \times 10^{-1}$ & - & - \\
P5 & $7.33 \times 10^{-1}$ & - & - \\
P6 & $6.96 \times 10^{-2}$ & $1.29 \times 10^{-3}$ & $6.85 \times 10^{-5}$ \\
P7 & $1.30 \times 10^{-2}$ & $1.23 \times 10^{-3}$ & $7.74 \times 10^{-5}$ \\
\hline
\end{tabular}

et al. [3] presented the optical sensitivity for RTL quartz that the peaks at $270^{\circ} \mathrm{C}$ and $305^{\circ} \mathrm{C}$ gave a high optical sensitivity while an insensitive optical property showed for the peak at $350^{\circ} \mathrm{C}$. This result supports the outcomes produced from the present study.

\section{Conclusion}

We investigated on the optical sensitivity of TL peaks separated by the CGCD method for RTL quartz. Using the $\mathrm{T}_{\mathrm{m}}-\mathrm{T}_{\text {stop }}$ method, we found the glow peak of RTL quartz measured in a temperature up to $450^{\circ} \mathrm{C}$ was overlapped with seven glow peaks. In addition, the general order kinetics model was appropriate to separate glow peaks. The optical sensitivity according to the exposure time to light from solar stimulation of the RTL signal for the dosimetric peaks exhibited only one exponential decay component for peak P4 and P5 and three different exponential decay components for peak P6 and P7.
The times taken to reduce the RTL signal to half of its initial value were 70 seconds for the peak $\mathrm{P} 4,54$ seconds for the peak P5, 9,840 seconds for the peak P6 and 26,580 seconds for the peak P7, respectively. From this result, we conclude that the optical sensitivity of peaks $\mathrm{P} 4$ and $\mathrm{P} 5$ gives much higher than that of peaks P6 and P7.

\section{Acknowledgements}

This research was supported by 2016 Research Grant from Kangwon National University (No. 520160283) and Basic Science Research Program through the National Research Foundation of Korea (NRF) funded by the Ministry of Education, Science and Technology (NRF-2017R1D1A1B03029608).

\section{References}

1. Franklin AD, Prescott JR, Robertson GB. Comparison of blue and red TL from quartz. Radiat. Meas. 2000;32(5-6):633-639.

2. Fattahi M, Stokes S. Extending the time range of luminescence dating using red TL (RTL) from volcanic quartz. Radiat. Meas. 2005;32(5-6):479-485.

3. Scholefield RB, Prescott JR. The red thermoluminescence of quartz: 3-D spectral measurement. Radiat. Meas. 1999;30(1):8395.

4. Lai Z, Murray A. Red TL of quartz extracted from Chinese loess: 
bleachability and saturation dose. Radiat. Meas. 2006;41(7-8): 836-840.

5. Pagonis V, Tatsis E, Kitis G. Search for common characteristics in the glow curves of quartz of various origins. Radiat. Prot. Dosim. 2002;100(1-4):373-376.

6. Yazici AN, Topaksu M. The analysis of thermoluminescence glow peaks of unannealed synthetic quartz. J. Phys. D: Appl. Phys. 2003; 36(6):620-627.

7. Horowitz YS, Yossian, D. Computerised glow curve deconvolution: application to thermoluminescence dosimetry. Radiat. Prot. Dosim. 1995;60:1-111.

8. Hashimoto T, Sugai N, Sakaue H, Yasuda K, Shirai N. Thermoluminescence (TL) spectra from quartz grains using on-line TLspectrometric system. Geochem. J. 1997;31(4):189-201.

9. Kitis G, Pagonis V, Drupieski C. Cooling rate effects on the thermoluminescence glow curves of Arkansas quartz. Phys. Status
Solidi A. 2003;198(2):312-321.

10. Song KW, Kim KB, Hong DG. Determination of trap parameters for thermoluminescence glow peaks of red thermoluminescence of quartz from Japan. Radiat. Eff. Defects Solids. 2010;165(4): 305-312.

11. McKeever SWS. On the analysis of complex thermoluminescence glow-curves: resolution into individual peaks. Phys. Status Solidi A. 1980;62(1):331-340.

12. Kitis G, Gomez-Ros JM, Tuyn JWN. Thermoluminescence glowcurve deconvolution functions for first, second and general orders of kinetics. J. Phys. D: Appl. Phys. 1998;31(19):2636-2641.

13. Balian HG, Eddy NW. Figure-of merit (FOM) an improved criterion over the normalized Chi-squared test of assessing goodness-of-fit of gamma-ray spectra peaks. Nucl. Instrum. Methods. 1997;145(2):389-395. 